\title{
Surgical management of "ab-extrinseco" main stem left coronary compression during pulmonary thromboendarterectomy
}

\author{
Sofia Martin-Suarez, Giulio Giovanni Cavalli, Gregorio Gliozzi, Carlo Mariani, Antonio Loforte, \\ Davide Pacini \\ Division of Cardiac Surgery, IRCCS Azienda Ospedaliero-Universitaria di Bologna, Bologna, Italy \\ Correspondence to: Sofia Marin-Suarez, MD, PhD. Department of Cardiac Surgery, S. Orsola Hospital, ALMA Mater Studiorum University of \\ Bologna, Via Massarenti n.9, 40138 Bologna, Italy. Email: docsofi74@hotmail.com; sofia.martinsuarez@aosp.bo.it.
}

Submitted Nov 14, 2021. Accepted for publication Dec 14, 2021.

doi: 10.21037/acs-2021-pte-21

View this article at: https://dx.doi.org/10.21037/acs-2021-pte-21

\section{Introduction}

Extrinsic compression of the left main coronary artery (LMCA) is a common but often underestimated complication of severe pulmonary hypertension $(\mathrm{PH})$ and resulting pulmonary artery $(\mathrm{PA})$ dilatation.

Chronic thromboembolic pulmonary hypertension $(\mathrm{CTEPH})$ is a rare cause of $\mathrm{PH}$, with a challenging diagnosis considering the ambiguous clinical presentation, especially when the patient's only complaint is angina due to PA enlargement. Diagnosis of extrinsic LMCA compression demands a high index of suspicion, because these patients usually have no relevant past medical history or risk factors for coronary artery disease.

Although computed tomography (CT) and cardiac magnetic resonance imaging may be preferred first-line, noninvasive approaches, diagnosis is eventually made with coronary angiography and intravascular ultrasound. These typically show LMCA ostial stenosis, usually without associated atherosclerosis: LMCA presents as an eccentric stenosis and is sharply displaced towards the left coronary sinus. Clinically significant dislocation of the LMCA due to a dilated PA main trunk is typically associated with an LMCA take-off angle $<60^{\circ}$ and a PA diameter more than four centimeters (1).

Despite no official consensus on the best treatment for this secondary cause of angina, whenever possible, treatment of the underlying cause of $\mathrm{PH}$ is helpful. Generally, percutaneous coronary intervention is considered firstchoice treatment, given the high surgical risk of patients with precapillary $\mathrm{PH}$.
If surgical correction of the primary cause of $\mathrm{PH}$ is possible, as in the case of CTEPH with pulmonary thromboendarterectomy (PTE), the postoperative reduction in PH is often sufficient to reduce compression of the LMCA. Nonetheless, if the PA is extremely enlarged, plication of its main trunk helps reduce LMCA compression (2). Conversely, should coronary angiography detect a critical stenosis, coronary artery bypass grafting (CABG) is considered.

\section{Clinical vignette}

A 60-year-old male was admitted to the emergency department after an episode of syncope. He complained of dyspnea and unrecognized angina that had worsened in the previous months. CT confirmed CTEPH with a critical extrinsic compression of the LMCA by the enlarged PA (maximum diameter $45 \mathrm{~mm}$ ). The patient was subsequently referred to our department and routine examinations were conducted including echocardiography, coronary and pulmonary angiography and right heart catheterization (RHC). These revealed severe precapillary PH [mean pulmonary artery pressure (mPAP) $48 \mathrm{mmHg}$, pulmonary capillary wedge pressure (PCWP) $8 \mathrm{mmHg}$, pulmonary vascular resistance (PVR) $4.3 \mathrm{WU}$ ] due to chronic, bilateral, segmental thromboembolism.

After 3 months of optimal but ineffective antihypertensive and anticoagulation therapy, the patient was considered eligible for surgery by our CTEPH multidisciplinary team. 


\section{Surgical technique}

\section{Preparation and exposition}

Routine intraoperative monitoring includes body temperatures (skin, bladder and nasopharyngeal temperature), bilateral invasive blood pressure, central venous pressure, Swan-Ganz parameters (PAP, PCWP, cardiac output, PVR, mixed venous oxygen saturation) and transesophageal echocardiography.

Following our standard approach, a full median sternotomy is performed with an oscillating saw to avoid opening the pleural cavities and to reduce the risk of pleural effusion.

Aorto-bicaval cannulation for cardiopulmonary bypass (CBP) is then instituted: notably, the superior venous cannula is positioned through the right atrial appendage to obtain optimal exposure of the right PA. Once the left ventricle and pulmonary trunk are drained, systemic cooling is started to reach deep hypothermia at 20 degrees Celsius. The aorta is cross-clamped at 25-26 degrees and single-dose crystalloid histidine-tryptophan-ketoglutarate cardioplegia (HTK-Custodiol; Koehler Chemi, AlsbachHaenlien, Germany) is delivered.

\section{Operation}

A longitudinal arteriotomy of the right PA is performed. Finding the cleavage plane is crucial to avoid damaging the arterial wall. The fibrotic, chronic thrombus is dissected from the wall and progressively removed. Care must be taken not to shatter the thrombus, because levering on it is crucial to perform the endarterectomy of the most distal branches including the subsegmental ones. During the procedure, 20 minutes of deep hypothermic circulatory arrest (DHCA) at 20 degrees Celsius is feasible and can be interrupted by 10 minutes of isothermal reperfusion if necessary (3). The purpose of adopting DHCA is to abolish the retrograde flow, which may impede a complete distal dissection of thrombi (4).

When a satisfactory PTE is achieved, the artery is reconstructed, and the same steps are carried out for the left PA. In consideration of this patient's PA dilatation, while rewarming, the trunk of the PA was reduced by linear plication and reinforced with two strips of Teflon felt, using two running $5 / 0$ polypropylene sutures.

Finally, weaning from CPB is a critical moment, often requiring inotropic support or mechanical circulatory support because of residual $\mathrm{PH}$ and temporary right ventricular failure (5).

\section{Post-operative outcome}

Post-operative observation is focused on cardiac output, peripheral and pulmonary pressures and resistances. Provided no major bleeding occurs, anticoagulation is started 6 hours after intensive care unit arrival with intravenous heparin and target aPTT ratio of two. On postoperative day five, oral anticoagulation is started in association with low molecular weight heparin (LMWH) until the INR is $>2.5$. Ceftazidime, a third-generation cephalosporin, is infused as empiric pulmonary antibiotic prophylaxis during the first seven postoperative days.

The patient's postoperative course was uneventful and he was discharged 19 days after surgery and was asymptomatic six months later. A significative hemodynamic improvement was measured with RHC (mPAP $35 \mathrm{mmHg}$, PVR $3.1 \mathrm{WU}$ ). A CT scan showed a reduced PA trunk diameter (maximum diameter $36 \mathrm{~mm}$ ) without compression of the LMCA.

\section{Comments}

CTEPH is the only cause of $\mathrm{PH}$ with a curative surgical treatment other than lung transplantation. However, not every patient is suitable for surgery so a multidisciplinary evaluation is mandatory. Moreover, extrinsic compression of the LMCA and related angina may complicate and delay the diagnostic and therapeutic decision process.

Generally, available treatment options for symptomatic extrinsic compression of LMCA are either percutaneous or surgical. Considering the high-risk profile of these patients, mainly related to their pre-capillary $\mathrm{PH}$, percutaneous revascularization has an advantageous risk-to-benefit ratio and is considered the first-line treatment (1).

If surgical treatment of CTEPH is feasible, PTE and the resulting drop of pulmonary pressure resolve the angina. However, when long-term PH causes extreme PA dilatation and persistent angina, linear plication of its main trunk should be considered during PTE. Finally, CABG is only considered when a critical atherosclerotic stenosis is discovered with coronary angiography.

\section{Acknowledgments}

Funding: None. 


\section{Footnote}

Conflicts of Interest: The authors have no conflicts of interest to declare.

Open Access Statement: This is an Open Access article distributed in accordance with the Creative Commons Attribution-NonCommercial-NoDerivs 4.0 International License (CC BY-NC-ND 4.0), which permits the noncommercial replication and distribution of the article with the strict proviso that no changes or edits are made and the original work is properly cited (including links to both the formal publication through the relevant DOI and the license). See: https://creativecommons.org/licenses/by-nc-nd/4.0/.

\section{References}

1. Galiè N, Saia F, Palazzini M, et al. Left Main Coronary Artery Compression in Patients With Pulmonary

Cite this article as: Martin-Suarez S, Cavalli GG, Gliozzi G, Mariani C, Loforte A, Pacini D. Surgical management of "ab-extrinseco" main stem left coronary compression during pulmonary thromboendarterectomy. Ann Cardiothorac Surg 2022;11(2):189-191. doi: 10.21037/acs-2021-pte-21
Arterial Hypertension and Angina. J Am Coll Cardiol 2017;69:2808-17.

2. Lee MS, Oyama J, Bhatia R, et al. Left main coronary artery compression from pulmonary artery enlargement due to pulmonary hypertension: a contemporary review and argument for percutaneous revascularization. Catheter Cardiovasc Interv 2010;76:543-50.

3. Jamieson SW, Kapelanski DP, Sakakibara N, et al. Pulmonary endarterectomy: experience and lessons learned in 1,500 cases. Ann Thorac Surg 2003;76:1457-62; discussion 1462-4.

4. Martin-Suarez S, Gliozzi G, Loforte A, et al. Pulmonary thrombo-endarterectomy for chronic thromboembolic pulmonary artery hypertension. Curr Chall Thorac Surg 2021;3:13.

5. Martin-Suarez S, Gliozzi G, Fiorentino M, et al. Role and management of extracorporeal life support after surgery of chronic thromboembolic pulmonary hypertension. Ann Cardiothorac Surg 2019;8:84-92. 